\title{
Development of a new positron counting system with SiPM readout for muon spin spectrometers
}

\author{
Sohtaro Kanda* for the MuSEUM collaboration \\ Department of Physics, University of Tokyo, 7-3-1 Hongo, Bunkyo-ku, Tokyo, 113-0033, Japan \\ E-mail: kandadpost.kek.jp
}

\begin{abstract}
Muon spin spectroscopy has been utilized for both particle physics and material science. At JPARC (Japan Proton Accelerator Research Complex), the world's highest-intensity pulsed muon beam has been realized. With the increase of the beam intensity, the requirement for the detector's high-rate capability becomes essential. Spectroscopy of muon spin is performed by measurement of positron angular asymmetry from muon decays. A new muon spin spectrometer with silicon photomultiplier (SiPM) readout is under development for precision measurement of muonium hyperfine splitting. The positron detector consists of tiled plastic scintillators, SiPMs, and fast readout-circuits with ASIC based ASD and FPGA implemented multi-hit TDC. A prototype of the detector was developed and its performance was evaluated with a high intensity pulsed muon beam at J-PARC. Based on an understanding of SiPM's characteristics and analog circuit response, a realistic event generator was developed for evaluation of systematic uncertainties of the measurement. Several methods of pileup correction were studied to minimize the systematic uncertainty. In this paper, overview of the project and details of the detector development are discussed.
\end{abstract}

International Conference on New Photo-detectors, PhotoDet 2015

6-9 July 2015

Moscow, Troitsk,Russia

\footnotetext{
* Speaker.
} 


\section{Introduction}

\subsection{Muon spin spectroscopy}

Muons are obtained from parity violating decay of pion with spin polarized along the direction of its momentum. The muon decay is also a parity violating process, so the averaged muon spin can be obtained by the measurement of the decay positron angular asymmetry.

In the presence of a magnetic field, the muon spin undergoes precession with a frequency proportional to the magnetic field strength. A non-uniformity or a distribution in the magnetic field causes muon spin relaxation. The muon stopped in matter is utilized as a local magnetic probe. This experimental technique is called muon spin rotation/relaxation/resonance $(\mu \mathrm{SR})$, which is known as an unique method to investigate the magnetic properties of a material and the dynamics of the magnetism in micro-second time scale. Furthermore, test of the standard model of particle physics and search for physics beyond the standard model can be performed via spin spectroscopy of muon or its bound state.

Figure 1 shows a schematic view of a muon spin spectrometer for the measurements of muon decay timing and positron emission angle. The incident positive muon beam is spin polarized in the upstream direction. A muon is implanted into a target material and the muon loses its kinetic energy via the electromagnetic interaction. The muon decays with a $2.2 \mu$ s lifetime and a positron is emitted with an angular asymmetry as a function of the positron kinetic energy. The decay positron is detected by a positron counter, which typically consists of a plastic scintillator and a photo multiplier tube (PMT). The time dependent angular asymmetry of positron counting contains the information of time evolution of muon spin. The positron counters are located upstream and downstream of the muon decay volume. The asymmetry is defined as $A=\frac{N_{F}-N_{B}}{N_{F}+N_{B}}$ where $N_{F}$ and $N_{B}$ are the number of positron detected by the forward/backward counter.

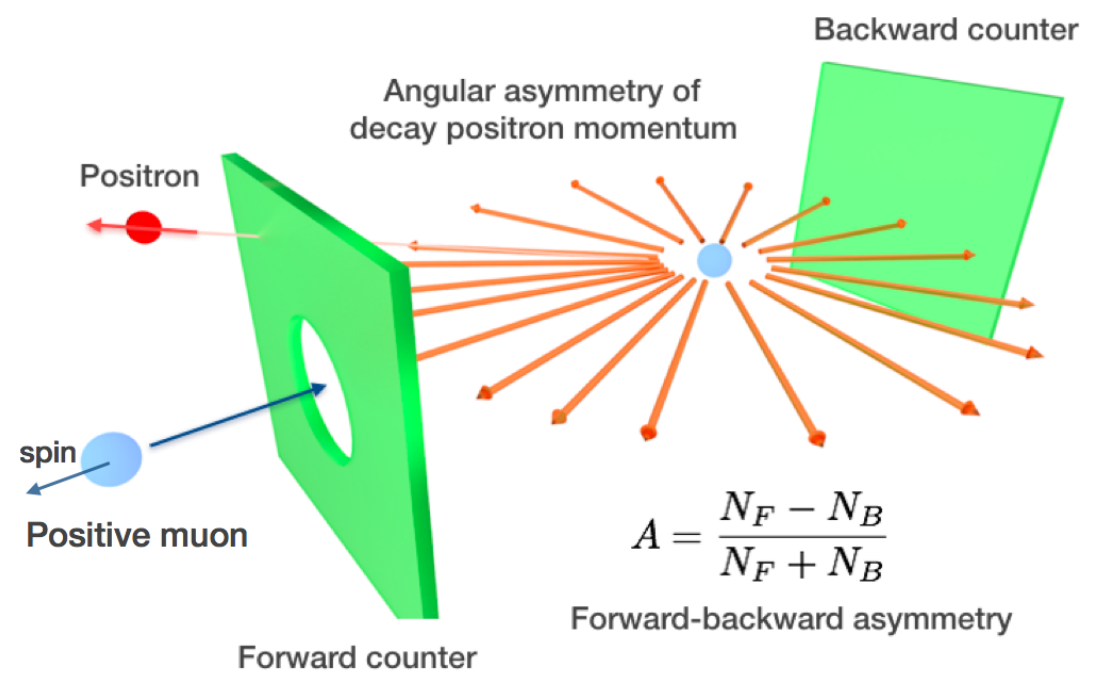

Figure 1: Schematic of a muon spin spectrometer

\subsection{Requirements for a muon spin spectrometer}

Muon spin spectroscopy can be performed with both continuous and pulsed muon beam. A 
pulsed muon beam is suitable for high statistical precision in a short measurement time. The world highest intensity pulsed muon beam was established at J-PARC Materials and Life Science Facility (MLF) Muon Science Establishment (MUSE). The typical muon beam intensity at MLF MUSE D-Line is $1 \times 10^{7} \mu^{+} / \mathrm{s}$ at $500 \mathrm{~kW}$ accelerator beam power. The beam is double pulsed in $600 \mathrm{~ns}$ interval with a repetition cycle of $25 \mathrm{~Hz}$. The high intensity pulsed muon beam has a beneficial effect on the accumulation of statistics in a limited beam time. On the other hand, the requirements for a positron counter is more severe than in the case of a continuous beam. A number of positrons are detected by a muon spin spectrometer at short intervals and signal pileup becomes severe. In order to achieve a large solid angle and the suppression of signal pileup at the same time, fine segmentation of the detector with an integrated readout electronics is essential.

\section{Detector design}

\subsection{Detector components}

A traditional muon spin spectrometer is the combination of plastic scintillator, PMT, and discrete signal processing electronics. To deal with the unprecedented high intensity pulsed muon beam at J-PARC, a new positron counting system for a muon spectrometer was designed. It consists of a small segment of plastic scintillator, a silicon photomultiplier (SiPM), and fast readout electronics with ASIC based amplifier, shaper, and discriminator (ASD) and multi-hit TDC implemented in FPGA.

\subsubsection{Plastic scintillator}

A plastic scintillator with a fast decay time constant and an emission wavelength around 450 $\mathrm{nm}$ is suitable for a new positron counter. EJ212 produced by ELGEN technologies was adopted. The dimension of a segment was decided based on simulation study as hereinafter described.

\subsubsection{SiPM}

Instead of a PMT, a silicon photomultiplier developed by Hamamatsu Photonics K.K. was adopted as a photo sensor. A MPPC (Multi Pixel Photon Counter) S12825-050P-01 was utilized, which has 667 pixels in $1.3 \mathrm{~mm} \times 1.3 \mathrm{~mm}$ active area $(50 \mu \mathrm{m}$ pixel pitch).

\subsubsection{Readout electronics}

As a series of readout electronics for SiPM, Kalliope (KEK Advanced Liner and Logic board Integrated Optical detector for Positron and Electron) has been developed by the Electronics Group and Institute of Materials Structure Science (IMSS) of KEK[四]. It consists of an analog signal circuit with an ASIC named VOLUME2012 and a digital circuit with a FPGA.

The ASIC contains two steps of voltage amplifier. Slow control parameters are described in 20 bits of register pattern for amplifier gain, signal shaping parameters related to amplifier biases, discriminator threshold, and fine tuning of the bias voltage for SiPM. The FPGA firmware contains the implementation of a multi-hit TDC with $1 \mathrm{~ns}$ timing resolution, which is realized by four phase rotating $250 \mathrm{MHz}$ clocks. 


\subsection{Muon spin spectrometer at J-PARC MUSE}

At J-PARC MLF MUSE, there were several proposals of muon spin spectrometer development for each specific purposes. For material science, general purpose detectors with three-axis Helmholtz coil and a variation for the measurement in high magnetic field have been developed[龱]. For particle physics, a new muon spin spectrometer was proposed for a precision microwave spectroscopy of muonium atom called MuSEUM (Muon Spectroscopy Experiment Using Microwave) experiment[[, []]. Following chapters describe in more details the positron counting system development for the MuSEUM experiment.

\section{Simulation study}

In order to estimate the positron counting rate and systematic uncertainties of the positron detector performance, a Monte-Carlo simulation framework was developed. The muon beam transportation and the particle tracking were simulated by a GEANT4-based event generator.

Figure 2 shows a geometrical simulation setup with an accumulated event display. The red lines correspond to the muon trajectories and the blue lines correspond to the positron trajectories. Three layers of positron counter were placed downstream of the gas chamber filled with krypton. A three-dimensional magnetic field distribution calculated by finite element method was implemented. Detector response was implemented based on the measurement results of a signal pulse waveform and photon yield. Individual fluctuation of SiPM's photo detection efficiency (PDE) was considered.

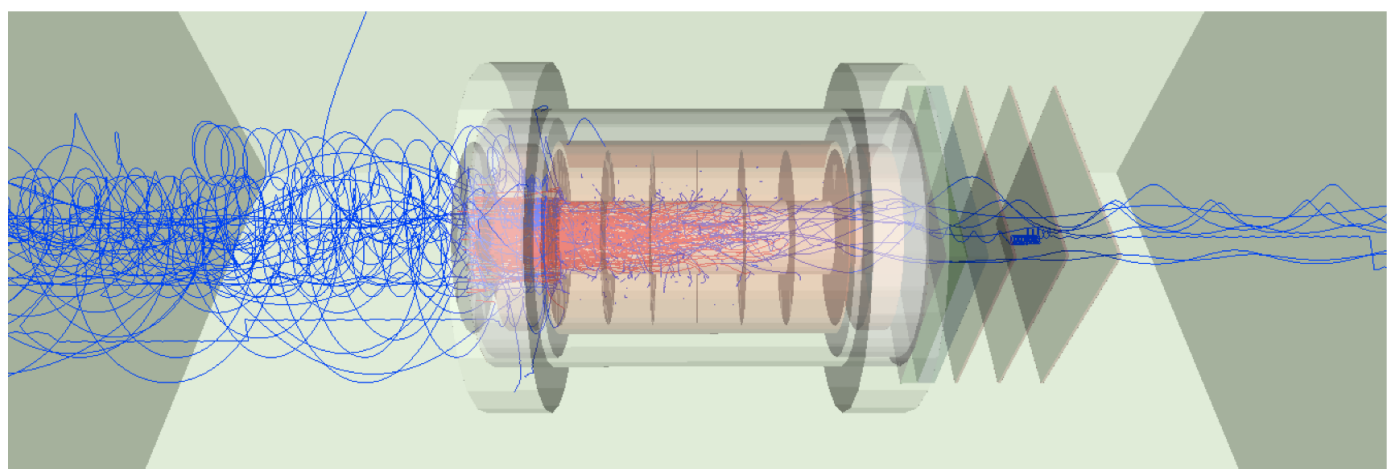

Figure 2: Simulation setup with an accumulated event display

Figure 3 shows a typical hit map on the detector. Figure 4 shows a simulated positron time spectrum. The blue line represents the ideal time spectrum while the red line represents the time spectrum with signal pileup.

The event loss due to the signal pileup was evaluated as a function of the maximum event rate. Figure 5 shows a simulated result of event loss. The ordinate axis indicates the number of lost events per total events. Polynomial fitting was performed and a correction function was obtained. Pileup correction was applied to the time spectrum for suppression of systematic uncertainty. Figure 6 presents a result of pileup correction where the abscissa axis indicates the relative statistics normalized by the total number of events. The black histogram is a result without pileup correction. The red histogram corresponds to a corrected result according to the correction function obtained 

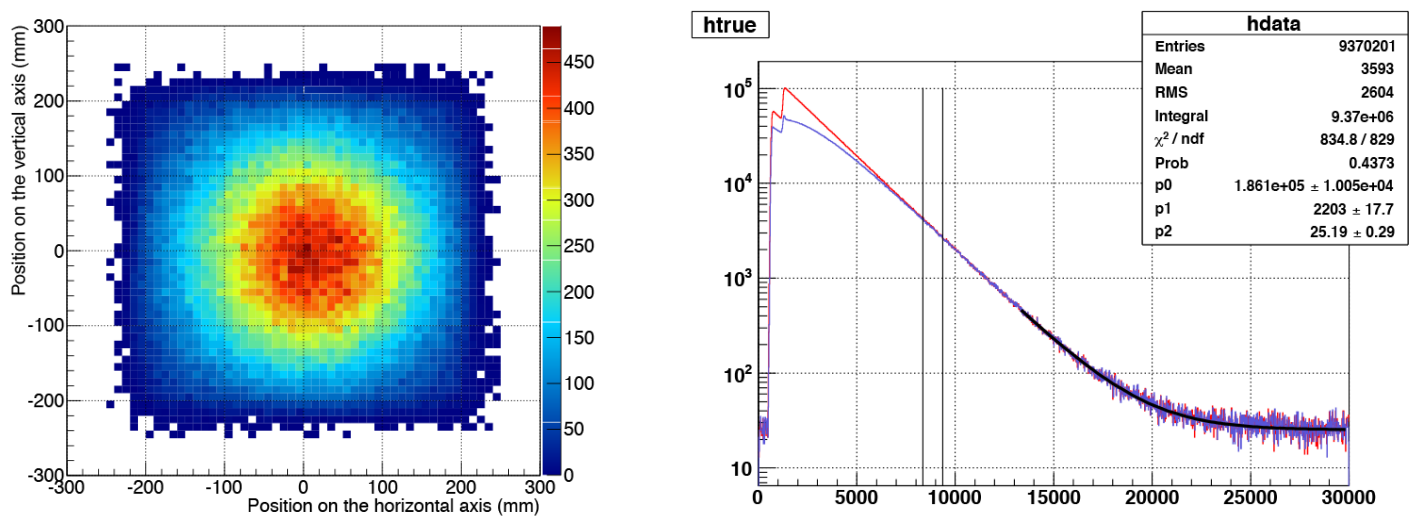

Figure 3: Simulated hit map on the detector plane

Figure 4: Simulated positron detection time spectrum

from Figure 5. After pileup correction, systematic uncertainty due to pileup was suppressed to an acceptable level.

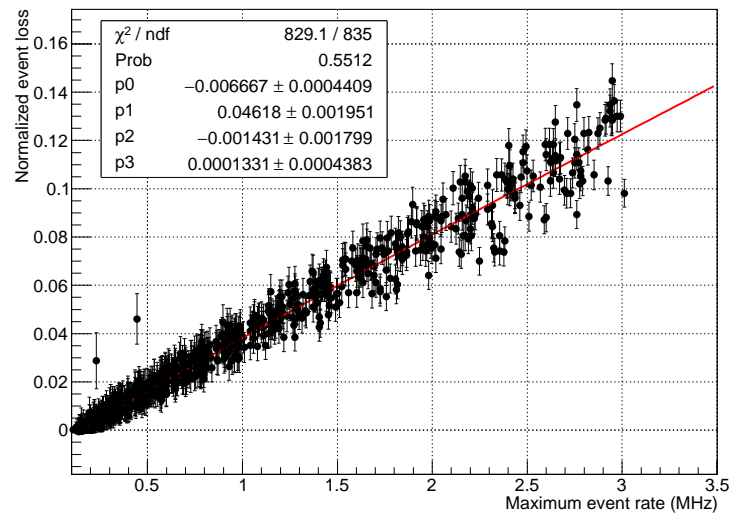

Figure 5: Simulated event loss

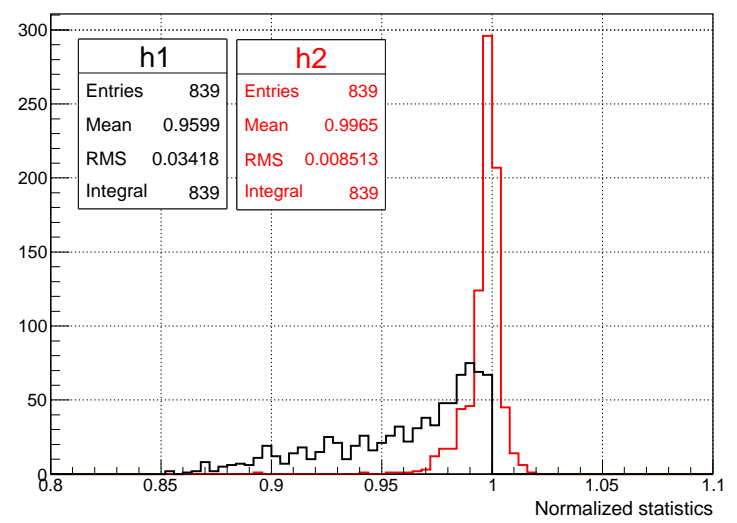

Figure 6: Simulated pileup correction

\section{Development of a positron counter}

\subsection{Designing and production of the detector}

For the proof of principle and the specification evaluation of a detector with plastic scintillator and SiPM, a detector prototype was developed and evaluation tests were performed[四]. A new muon spin spectrometer for the MuSEUM experiment was developed on the ground of $t$ heprototype evaluation and simulation study. Figure 7 is a conceptual design of the detector. An unit cell of the positron counter consists of a $10 \mathrm{~mm} \times 10 \mathrm{~mm} \times 3 \mathrm{~mm}$ plastic scintillator with a SiPM directly attached to the center of the detector segment. Figure 8 is a photograph of the developed detector plane with scintillator segments. The SiPMs were arranged in a matrix on a PCB (Printed Circuit Board) plane.

\subsection{Operation and tuning of the detector}

With the objective of high-rate data processing, Kalliope readout system has no ADC for pulse height or charge measurement. In order to perform gain tuning and operation monitoring, 


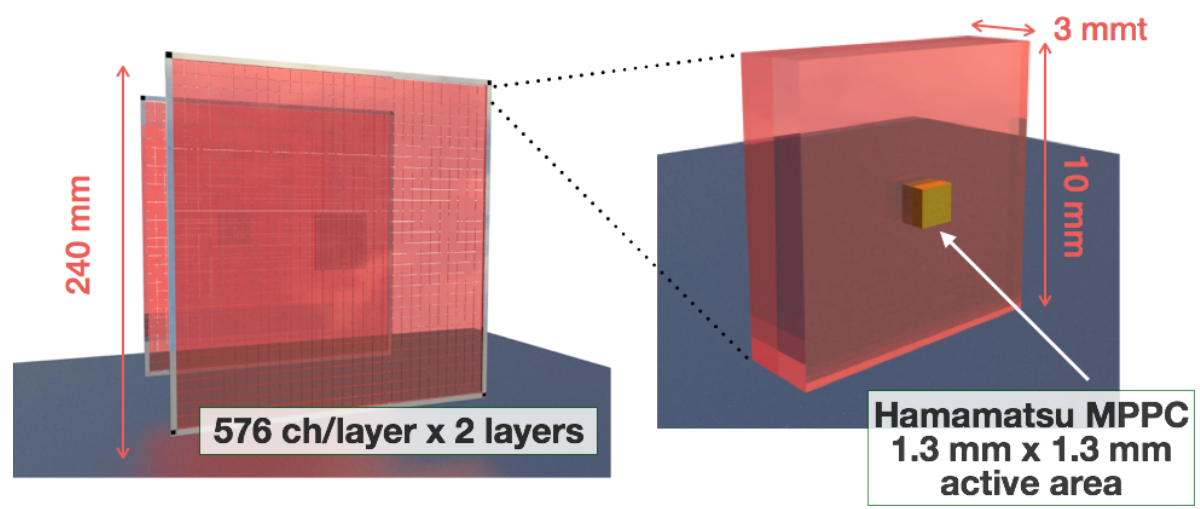

Figure 7: Conceptual design of the detector

time over threshold (TOT) sampling was implemented in the FPGA firmware. Figure 9 shows a typical measured TOT spectrum with discrete structure derived from the Geiger mode operation of the SiPM. Due to a correlation between TOT and signal pulse height, uniform performance of the detector is ensured by tuning the operation voltages for each SiPMs.

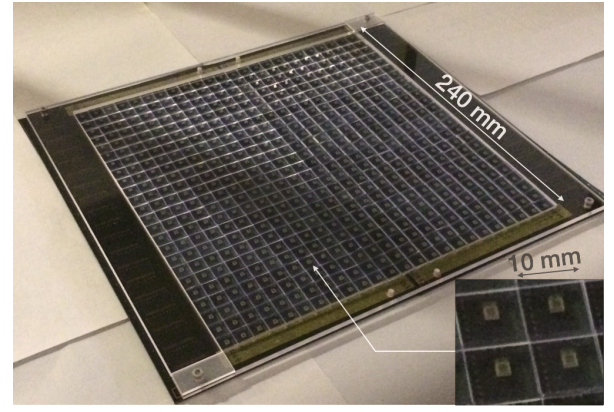

Figure 8: Developed detector plane

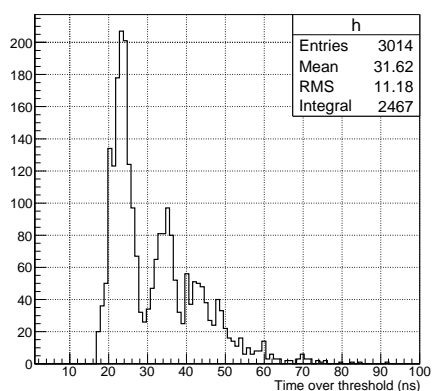

Figure 9: Measured TOT spectrum

\section{Summary}

Muon spin spectroscopy is an unique technique for both material science and particle physics. For muon spin spectroscopy with high intensity pulsed muon beam, development of a new type of highly segmented positron counter was proposed. As a solution that meets the requirements for positron counting system, a positron counter with segmented plastic scintillator, $\mathrm{SiPM}$, and fast readout electronics was designed. After prototype development and simulation study, a high rate capable positron counter for the MuSEUM experiment was developed. The detector system was assembled and a beam test is scheduled in November 2015 at J-PARC MLF MUSE. This work was supported by JSPS Grant-in-Aid for JSPS Fellows Number 26-11374.

\section{References}

[1] K. M. Kojima et al., J. Phys. Conf. Ser. 551, 012063 (2014).

[2] K. Shimomura, AIP Conf. Proc. 1382, 245 (2011).

[3] P. Strasser et al., PoS(NUFACT2014)087 (2015).

[4] S. Kanda et al., JPS Conf. Proc. 8, 025006 (2015). 